\title{
Subquadratic Submodular Function Minimization
}

\author{
Deeparnab Chakrabarty* $\quad$ Yin Tat Lee $^{\dagger} \quad$ Aaron Sidford ${ }^{\ddagger} \quad$ Sam Chiu-wai Wong $\S$
}

November 1, 2016

\begin{abstract}
Submodular function minimization (SFM) is a fundamental discrete optimization problem which generalizes many well known problems, has applications in various fields, and can be solved in polynomial time. Owing to applications in computer vision and machine learning, fast SFM algorithms are highly desirable. The current fastest algorithms [36] run in $O\left(n^{2} \log n M \cdot \mathrm{EO}+n^{3} \log { }^{O(1)} n M\right)$ time and $O\left(n^{3} \log ^{2} n \cdot \mathrm{EO}+n^{4} \log { }^{O(1)} n\right)$ time respectively, where $M$ is the largest absolute value of the function (assuming the range is integers) and EO is the time taken to evaluate the function on any set. Although the best known lower bound on the query complexity is only $\Omega(n)$ [23], the current shortest non-deterministic proof [10] certifying the optimum value of a function requires $\Omega\left(n^{2}\right)$ function evaluations.

The main contribution of this paper are subquadratic SFM algorithms. For integer-valued submodular functions, we give an SFM algorithm which runs in $O\left(n M^{3} \log n \cdot \mathrm{EO}\right)$ time giving the first nearly linear time algorithm in any known regime. For real-valued submodular functions with range in $[-1,1]$, we give an algorithm which in $\tilde{O}\left(n^{5 / 3} \cdot \mathrm{EO} / \varepsilon^{2}\right)$ time returns an $\varepsilon$-additive approximate solution. At the heart of it, our algorithms are projected stochastic subgradient descent methods on the Lovasz extension of submodular functions where we crucially exploit submodularity and data structures to obtain fast, i.e. sublinear time subgradient updates. The latter is crucial for beating the $n^{2}$ bound - we show that algorithms which access only subgradients of the Lovasz extension, and these include the empirically fast Fujishige-Wolfe heuristic [48, 15] and the theoretically best cutting plane methods [36] , must make $\Omega(n)$ subgradient calls (even for functions whose range is $\{-1,0,1\}$ ).
\end{abstract}

\footnotetext{
*Microsoft Research, India. Email: deeparnab@gmail.com

$\dagger$ Microsoft Research, Email: yile@microsoft.com

¥Stanford University. Email: sidford@stanford.edu

$\S$ UC Berkeley. Email: samcwong@berkeley.edu
} 


\section{Introduction}

Submodular functions are set functions that prescribe a value to every subset of a finite universe $U$ and have the following diminishing returns property: for every pair $S \subseteq T \subseteq U$, and for every element $i \notin T$, $f(S \cup i)-f(S) \geq f(T \cup i)-f(T)$. Such functions arise in many applications. For instance, the utility functions of agents in economics are often assumed to be submodular, the cut functions in directed graphs or hypergraphs are submodular, the entropy of a given subset of random variables is submodular, etc. Submodular functions have been extensively studied for more than five decades [9, 11, 39, 16, 40].

One of the most important problems in this area is submodular function minimization (SFM, henceforth) which asks to find the set $S$ minimizing $f(S)$. Note that submodular functions need not be monotone and therefore SFM is non-trivial. In particular, SFM generalizes the minimum cut problem in directed graphs and hypergraphs, and is a fundamental problem in combinatorial optimization. More recently, SFM has found many applications in areas such as image segmentation [5, 32, 33] and speech analysis [37, 38]. Owing to these large scale problems, fast SFM algorithms are highly desirable.

We assume access to an evaluation oracle for the submodular function, and use EO to denote the time taken per evaluation. An amazing property of submodular functions is that SFM can be exactly solved with polynomial many queries and in polynomial time. This was first established via the ellipsoid algorithm [21] in 1981, and the first polynomial combinatorial algorithms were obtained [10, 25, 45, 26] much later.

The current fastest algorithms for SFM are by the second, third, and fourth authors of this paper [36] who give $O\left(n^{2} \log n M \cdot \mathrm{EO}+n^{3} \log ^{O(1)} n M\right)$ time and $O\left(n^{3} \log ^{2} n \cdot \mathrm{EO}+n^{4} \log ^{O(1)} n\right)$ time algorithms for SFM. Here $M$ is the largest absolute value of the integer-valued function. The former running time is a (weakly) polynomial running time, i.e. it depends polylogarithmically on $M$, while the latter is a strongly polynomial running time, i.e. it does not depend on $M$ at all. Although good in theory, known implementations of the above algorithms are slow in practice [17, 18, 1, 8]. A different algorithm, the so-called Fujishige-Wolfe algorithm $[48,15]$ seems to have the best empirical performance $[1,31,3]$ among general purpose SFM algorithms. Recently the Fujishige-Wolfe algorithm and variants were shown $[8,35]$ to run in $O\left(\left(n^{2} \cdot \mathrm{EO}+n^{3}\right) M^{2}\right)$ time, proving them to be pseudopolynomial time algorithms, that is having running time $O(\operatorname{poly}(n, \mathrm{EO}, M))$.

In this paper we also consider approximate SFM. More precisely, for submodular functions whose values are in the range $[-1,+1]$ (which is without loss of generality by scaling), we want to obtain additive approximations $^{1}$, that is, return a set $S$ with $f(S) \leq$ opt $+\varepsilon$. Although approximate SFM has not been explicitly studied before, previous works [36, 1, 8] imply $O\left(n^{2} \mathrm{EO} \log ^{O(1)}(n / \varepsilon)\right)$-time and $O\left(\left(n^{2} \cdot \mathrm{EO}+n^{3}\right) / \varepsilon^{2}\right)$-time algorithms. Table 1 summarizes the above discussion.

\begin{tabular}{|c||c|c|c|}
\hline Regime & Previous Best Running Time & Our Result & Techniques \\
\hline \hline Strongly Polynomial & \multicolumn{2}{||}{$O\left(n^{3} \log ^{2} n \cdot \mathrm{EO}+n^{4} \log ^{O(1)} n\right)[36]$} & Cutting Plane + Dimension Collapsing \\
\hline Weakly Polynomial & \multicolumn{2}{|c|}{$O\left(n^{2} \log n M \cdot \mathrm{EO}+n^{3} \log ^{O(1)} n M\right)[36]$} & Cutting Plane \\
\hline Pseudo Polynomial & $O\left(\left(n^{2} \cdot \mathrm{EO}+n^{3}\right) M^{2}\right)[8,35]$ & $O\left(n M^{3} \cdot \mathrm{EO}\right)$ & See Section 1.2 \\
\hline$\varepsilon$-Approximate & $O\left(n^{2} \cdot \mathrm{EO} / \varepsilon^{2}\right)[8,35,1]$ & $\tilde{O}\left(n^{5 / 3} \cdot \mathrm{EO} / \varepsilon^{2}\right)$ & See Section 1.2 \\
\hline
\end{tabular}

Table 1: Running times for minimizing a submodular function defined on a universe of size $n$ that takes integer values between $-M$ and $M$ (except for $\varepsilon$-approximate algorithms we assume the submodular function is real-valued with range in $[-1,1])$. EO denotes the time to evaluate the submodular function on a set.

In particular, the best known dependence on $n$ is quadratic even when the exact algorithms are allowed to be pseudopolynomial, or when the $\varepsilon$-approximation algorithms are allowed to have a polynomial dependence on $\varepsilon$. This quadratic dependence seems to be a barrier. For exact SFM, the smallest known non-deterministic proof $[11,10]$ that certifies optimality requires $\Theta\left(n^{2}\right)$ queries, and even for the approximate case, nothing

\footnotetext{
${ }^{1}$ We also show in Appendix A how to obtain a multiplicative approximation under a mild condition on $f$. Such a condition is necessary as multiplicative approximation is ill defined in general.
} 
better is known (see Appendix D). Furthermore, in this paper we prove that a large class of algorithms which includes the Fujishige-Wolfe algorithm[48, 15] and the cutting planealgorithms of Lee et al.[36], as stated need to make $\Omega\left(n^{2}\right)$ queries. More precisely, these algorithms do not exploit the full power of submodularity and work even with the weaker model of having access only to the "subgradients of the Lovasz Extension" where each subgradient takes $\Theta(n)$ queries. We prove that any algorithm must make $\Omega(n)$ subgradient calls implying the quadratic lower bound for this class of algorithms. Furthermore, our lower bound holds even for functions with range $\{-1,0,1\}$, and so trivially the lower bound also holds for approximate SFM as well.

\subsection{Our Results}

In this paper, we describe exact and approximate algorithms for SFM which run in time subquadratic in the dimension $n$. Our first result is a pseudopolynomial time exact SFM algorithm with nearly linear dependence on $n$. More precisely, for any integer valued submodular function with maximum absolute value $M$, our algorithm returns the optimum solution in $O\left(n M^{3} \log n \cdot \mathrm{EO}\right)$ time. This has a few consequences to the complexity theory of SFM. First, this gives a better dependence on $n$ for pseudopolynomial time algorithm. Second, this shows that to get a super-linear lower bound on the query complexity of SFM, one need to consider a function with super constant function values. ${ }^{2}$ Third, this completes the following picture on the complexity of SFM: the best known strongly polynomial time algorithms have query complexity $\tilde{O}\left(n^{3}\right)$, the best known (weakly) polynomial time algorithms have query complexity $\tilde{O}\left(n^{2}\right)$, and our result implies the best pseudopolynomial time algorithm has query complexity $\tilde{O}(n)$.

Our second result is a subquadratic approximate $S F M$ algorithm. More precisely, we give an algorithm which in $\tilde{O}\left(n^{5 / 3} \mathrm{EO} / \varepsilon^{2}\right)$ time, returns an $\varepsilon$-additive approximate solution. To break the quadratic barrier, that arise from the need to compute $\Omega(n)$ subgradient each of which individully we do not know how to compute faster than $\Omega(n \cdot \mathrm{EO})$, we wed continuous optimization techniques with properties deduced from submodularity and simple data structures. These allow us to compute and use gradient updates in a much more economical fashion. We believe that that the ability to obtain subquadratic approximate algorithms for approximate submodular minimization is an interesting structural result that could have further implications. ${ }^{3}$

Finally, we show how to improve upon these results further if we know that the optimal solution is sparse. This may be a regime of interest for certain applications where the solution space is large (e.g. structured predictions have exponentially large candidate sets [44]), and as far as we are aware, no other algorithm gives sparsity-critical results.

\subsection{Overview of Techniques}

In a nutshell, all are our algorithms are projected, stochastic subgradient descent algorithms on the Lovasz extension $\hat{f}$ of a submodular function with economical subgradient updates. The latter crucially uses submodularity and serves as the point of departure from previous black-box continuous optimization based methods. In this section, we give a brief overview of our techniques.

The Lovasz extension $\hat{f}$ of a submodular function is a non-smooth convex function whose (approximate) minimizers leads to (approximate) SFM. Subgradient descent algorithms maintain a current iterate $x^{(t)}$ and take a step in the negative direction of a subgradient $g\left(x^{(t)}\right)$ at $x^{(t)}$ to get the next iterate $x^{(t+1)}$. In general, the subgradient of a Lovasz extension takes $O(n \mathrm{EO})$ to compute. As stated above, the $\Omega(n)$ lower bound on the number of iterations needed, implies that if we naively recompute the subgradients at every iterations, we cannot beat the quadratic barrier. Our main technical contribution is to exploit submodularity so that $g\left(x^{(t+1)}\right)$ can be computed in sublinear time given $x^{(t)}$ and $g\left(x^{(t)}\right)$.

The first implication of submodularity is the observation (also made by [30, 24]) that $\ell_{1}$-norms of the

\footnotetext{
${ }^{2}$ Conversely, [22, Thm 5.7] shows that we need at least $n$ queries of evaluation oracle to minimize a submodular function with range in $\{0,1,2\}$.

${ }^{3}$ Note that simple graph optimization problems, such as directed minimum $s$ - $t$ cut, is not one of these (See Appendix C).
} 
subgradients are bounded by $O(M)$ if the submodular function is in $[-M, M]$. When the function is integer valued, this implies that the subgradients are sparse and have only $O(M)$ non-zero entries. Therefore, information theoretically, we need only $O(M)$ bits to get $g\left(x^{(t+1)}\right)$ from $g\left(x^{(t)}\right)$. However, we need an algorithm to find the positions at which they differ. To do so, we use submodularity again. We observe that given any point $x$ and non-negative, $k$-sparse vector $e$, the difference vector $d:=g(x+e)-g(x)$ is non-positive at points corresponding to support of $e$ and non-negative everywhere else. Furthemore, on a "contiguous set" of coordinates, the sum of these entries in $d$ can be computed in $O(\mathrm{EO})$ time. Armed with this, we create a binary search tree (BST) type data structure to find the $O(M)$ non-zero coordinates of $d$ in $O(M \cdot \mathrm{EO} \log n)$ time (as opposed to $O(n \cdot \mathrm{EO})$ time). This, along with standard subgradient descent analysis yields our $O\left(n M^{3} \mathrm{EO} \log n\right)$-algorithm.

When the submodular function is real valued between $[-1,1]$, although the $\ell_{1}$-norm is small the subgradient can have full support. Therefore, we cannot hope to evaluate the gradient in sublinear time. We resort to stochastic subgradient descent where one moves along a direction whose expected value is the negative subgradient and whose variance is bounded. Ideally, we would have liked a fast one-shot random estimation of $g\left(x^{(t+1)}\right)$; unfortunately we do not how to do it. What we can do is obtain fast estimates to the difference vector $d$ mentioned above. As discussed above, the vector $d$ has $O(k)$ "islands" of non-negative entries peppered with $O(k)$ non-positive entries. We maintain a data-structure which with $O(k \mathrm{EO} \log n)$ preprocessing time can evaluate the sums of the entries in these islands in $O(\mathrm{EO} \log n)$ time. Given this, we can sample a coordinate $j \in[n]$ with probability proportional to $\left|d_{j}\right|$ in a similar time. Thus we get a random estimate of the vector $d$ whose variance is bounded by a constant.

To get the stochastic subgradient, however, we need to add these difference vectors and this accumulates the variance. To keep the variance in control, we run the final algorithm in batches. In each batch, as we progress we take more samples of the $d$-vector to keep the variance in check. This however increases the sparsity (the $k$ parameter), and one needs to balance the effects of the two. At the end of each batch, we spend $O(n \mathrm{EO})$ time computing the deterministic subgradient and start the process over. Balancing the number of iterations and length of batches gives us the $\tilde{O}\left(n^{5 / 3} \mathrm{EO} \varepsilon^{-2}\right)$-time algorithm for $\varepsilon$-approximate SFM.

\subsection{Related Work}

Submodularity, and indeed SFM, has a rich body of work and we refer the reader to surveys of Fujishige [16] and McCormick[40] for a more detailed pre-2006 version. Here we mention a few subsequent related works which were mostly inspired by application in machine learning.

Motivated by applications in computer vision [5,4] which require fast algorithms for SFM, researchers focused on minimization of decomposable submodular functions which are expressible as sum of "simple" submodular functions. It is assumed that simple submodular functions can be minimized fast (either in practice or in theory). Such a study was initiated by Stobbe and Krause [46] and Kolmogorov [34] who gave faster (than general SFM) algorithms for such functions. More recently, motivated by work of Jegelka et al. [29], algorithms with linear convergence rates [43, 12] have been obtained. That is, they get $\varepsilon$-approximate algorithms with dependence on $\varepsilon$ being $\log (1 / \varepsilon)$. .

We end our introductory discussion by mentioning the complexity of constrained SFM where one wishes to minimize over sets satisfying some constraints. In general constrained SFM is much harder than unconstrained SFM. For instance the minimum cut problem with cardinality constraints becomes the balanced partitioning problem which is APX-hard. More generally, Svitkina and Fleischer [47] show that a large class of constrained SFM problems cannot be approximated to better than $\tilde{O}(\sqrt{n})$ factors without making exponentially many queries. In contrast, Goemans and Soto [20] prove that symmetric submodular functions can be minimized over a large class of constraints. Inspired by machine learning applications, Iyer et al. [28, 27] give algorithms for a large class of constrained SFM problems which have good approximation guarantees if the curvature of the functions are small. 


\section{Preliminaries}

Here we introduce notations and general concepts used throughout this paper.

\subsection{General Notation}

We let $[n] \stackrel{\text { def }}{=}\{1, \ldots, n\}$ and $[0,1]^{n} \stackrel{\text { def }}{=}\left\{x \in \mathbb{R}^{n}: x_{i} \in[0,1] \forall i \in[n]\right\}$. Given a permutation $P=\left(P_{1}, \ldots, P_{n}\right)$ of $[n]$, let $P[j] \stackrel{\text { def }}{=}\left\{P_{1}, P_{2}, \ldots, P_{j}\right\}$ be the set containing the first $j$ elements of $P$. Any point $x \in \mathbb{R}^{n}$ defines the permutation $P_{x}$ consistent with $x$ where $x_{P_{1}} \geq x_{P_{2}} \geq \ldots \geq x_{P_{n}}$ with ties broken lexicographically. We denote by $\mathbf{1}_{i} \in \mathbb{R}^{n}$ the indicator vector for coordinate $i$, i.e. $\mathbf{1}_{i}$ has a 1 in coordinate $i$ and a 0 in all other coordinates. We call a vector $s$-sparse if it has at most $s$ non-zero entries.

\subsection{Submodular Functions}

Throughout this paper $f: 2^{U} \rightarrow \mathbb{R}$ denotes a submodular function on a ground set $U$. For notational convenience we assume without loss of generality that $U=[n]$ for some positive integer $n$ and that $f(\emptyset)=0$ (as this can be enforced by subtracting $f(\emptyset)$ from for the value of all sets while preserving submodularity). Recall that $f$ is submodular if and only if it obeys the property of diminishing marginal returns: for all $S \subseteq T \subseteq[n]$ and $i \notin T$ we have

$$
f(S \cup\{i\})-f(S) \geq f(T \cup\{i\})-f(T) .
$$

We let opt $\stackrel{\text { def }}{=} \min _{S \subseteq[n]} f(S)$ be the minimum value of $f$. We denote by EO the time it takes to evaluate $f$ on a set $S$. More precisely, we assume given a linked list storing a permutation $P$ of $[n]$, and a position $k$, we can evaluate $f(P[k])$ in $\mathrm{EO}$ time.

\subsection{The Lovasz Extension}

Our results make extensive use of the Lovasz extension, a convex, continuous extension of a submodular function to the interior of the $n$-dimensional hypercube, i.e. $[0,1]^{n}$.

Definition 1 (Lovasz Extension). Given a submodular function $f$, the Lovasz extension of $f$, denoted as $\hat{f}:[0,1]^{n} \rightarrow \mathbb{R}$, is defined for all $x \in[0,1]^{n}$ by $\hat{f}(x)=\sum_{j \in[n]}\left(f([P[j])-f(P[j-1])) x_{i_{j}}\right.$ where $P=P_{x}=$ $\left(P_{1}, \ldots, P_{n}\right)$ is the permutation consistent with $x$.

Note that since $f(\emptyset)=0$ this definition is equivalent to

$$
\hat{f}(x)=f(P[n]) x_{P_{n}}+\sum_{j \in[n-1]} f\left([P[j])\left(x_{P_{j}}-x_{P_{j+1}}\right) .\right.
$$

We make use of the following well known facts regarding submodular functions (see e.g. [39, 16]).

Theorem 2 (Lovasz Extension Properties). The following are true for all $x \in[0,1]^{n}$ :

- Convexity: The Lovasz extension is convex.

- Consistency: For $x \in\{0,1\}^{n}$ we have $\hat{f}(x)=f(S(x))$ where $S(x)=\left\{i \in S: x_{i}=1\right\}$.

- Minimizers: $\min _{x \in[0,1]^{n}} \hat{f}(x)=\min _{S \subseteq[n]} f(S)$. 
- Subgradients: The vector $g(x) \in \mathbb{R}^{n}$ defined by $g(x)_{P_{k}} \stackrel{\text { def }}{=} f(P[k])-f(P[k-1])$ is a subgradient of $\hat{f}$ at $x$, where $P=P_{x}$ is the permutation consistent with $x$. Let us call this the Lovasz subgradient.

We conclude with a few straightforward computational observations regarding the Lovasz extension and its subgradients. First note that for $x \in[0,1]^{n}$ we can evaluate $\hat{f}(x)$ or compute $g(x)$ in time $O(n \mathrm{EO}+n \log n)$ simply by sorting the coordinates of $f$ and evaluating $f$ at the $n$ desired sets. Also, note that by (2.1) the Lovasz extension evaluated at $x \in[0,1]^{n}$ is a non-negative combination of the value of $f$ at $n$ sets. Therefore computing the smallest of these sets gives a set $S \subseteq[n]$ such that $f(S) \leq \hat{f}(x)$ and we can clearly compute this, again in $O(n \mathrm{EO}+n \log n)$ time. Therefore for any algorithm which approximately minimizes the Lovasz extension with some (additive) error $\varepsilon$, we can always find a set $S$ achieving the same error on $f$ by just paying an additive $O(n \mathrm{EO}+n \log n)$ in the running time.

\subsection{Subgradient Descent}

Our algorithmic results make extensive use of subgradient descent (or mirror descent) and their stochastic analogs. Recall that for a convex function $h: \chi \rightarrow \mathbb{R}$, where $\chi \subseteq \mathbb{R}^{n}$ is a compact convex set, a vector $g \in \mathbb{R}^{n}$ is a subgradient of $h$ at $x \in \chi$ if for all $y \in \chi$ we have

$$
h(y) \geq h(x)+g^{\top}(y-x) .
$$

For such an $h$ we let $\partial h(x)$ denote the set of subgradients of $h$ at $x$. An algorithm that on input $x$ outputs $\tilde{g}(x) \in \partial h(x)$ is a subgradient oracle for $h$. Similarly, an algorithm that on input $x$ outputs a random $\tilde{g}(x)$ such that $\mathbf{E} \tilde{g}(x) \in \partial h(x)$ is a stochastic subgradient oracle for $h$.

One of our main algorithmic tools is the well known fact that given a (stochastic) subgradient oracle we can minimize a convex function $h$. Such algorithms are called (stochastic) subgradient descent algorithms and fall into a more general framework of algorithms known as mirror descent. These algorithms are very well studied and there is a rich literature on the topic. Below we provide one specific form of these algorithms adapted from [6] that suffices for our purposes.

Theorem 3 (Projected (Stochastic) Subgradient Descent ${ }^{4}$ ). Let $\chi \subseteq \mathbb{R}^{n}$ denote a compact convex set, $h: \chi \rightarrow \mathbb{R}$ be a convex function, $\tilde{g}$ be a (stochastic) subgradient oracle for which $\mathbf{E}\|\tilde{g}(x)\|_{2}^{2} \leq B^{2}$ for all $x \in \chi$, and $R^{2} \stackrel{\text { def }}{=} \sup _{x \in \chi} \frac{1}{2}\|x\|_{2}^{2}$. Now consider the iterative algorithm starting with

$$
x^{(1)}:=\operatorname{argmin}_{x \in \chi}\|x\|_{2}^{2}
$$

and for all $s$ we compute

$$
x^{(s+1)}:=\operatorname{argmin}_{x \in \chi}\left\|x-\left(x^{(s)}-\eta \tilde{g}\left(x^{(s)}\right)\right)\right\|_{2}^{2}
$$

Then for $\eta=\frac{R}{B} \sqrt{\frac{2}{t}}$ we have

$$
\mathbf{E} h\left(\frac{1}{t} \sum_{i \in[t]} x^{(s)}\right)-\min _{x \in \chi} h(x) \leq R B \sqrt{\frac{2}{t}} .
$$

We refer to this algorithm as projected stochastic subgradient descent when $\tilde{g}$ is stochastic and as projected subgradient descent when $\tilde{g}$ is deterministic, though we often omit the term projected for brevity. Note that when $\tilde{g}$ is deterministic the results are achieved exactly rather than in expectation.

\footnotetext{
${ }^{4}$ This is Theorem 6.1 from [6] restated where we used the "ball setup" with $\Phi(x)=\frac{1}{2}\|x\|_{2}^{2}$ so that $\mathcal{D}=\mathbb{R}^{n}$ and $D_{\Phi}(x, y)=$ $\frac{1}{2}\|x-y\|_{2}^{2}$. We also used that $\operatorname{argmin}_{x \in \chi} \eta g^{\top} x+\frac{1}{2}\left\|x-x_{t}\right\|_{2}^{2}=\operatorname{argmin}_{x \chi}\left\|x-\left(x_{t}-\eta g\right)\right\|_{2}^{2}$.
} 


\section{Faster Submodular Function Minimization}

In this section we provide faster algorithms for SFM. In particular we provide the first nearly linear time pseudopolynomial algorithm for SFM and the first subquadratic additive approximation algorithm for SFM. Furthermore, we show how to obtain even faster running times when the SFM instance is known to have a sparse solution.

All our algorithms follow the same broad algorithmic framework of using subgradient descent with a specialized subgradient oracle. Where they differ is in how the structure of the submodular functions is exploited in implementing these oracles. The remainder of this section is structured as follows: in Section 3.1 we provide the algorithmic framework we use for SFM, in Section 3.2, we prove structural properties of submodular functions that we use to compute subgradients, in Section 3.3, we describe our nearly linear time pseodopolynomial algorithms, in Section 3.4, we describe our subquadratic additive approximation algorithm, and in Section 3.5, we show how to improve these results when SFM has a sparse solution.

We make minimal effort to control logarithmic factors in through this section and note that some of the factors come from sorting and therefore maybe can be removed depending on the desired computational model.

\subsection{Algorithmic Framework}

All our algorithms for SFM follow the same broad algorithmic framework. We consider the Lovasz extension $\hat{f}:[0,1]^{n} \rightarrow \mathbb{R}$, and perform projected (stochastic) subgradient descent on $\hat{f}$ over the convex domain $\chi=[0,1]^{n}$. While the subgradient oracle construction differs between algorithms (and additional care is used to improve when the solution is sparse, i.e. Section 3.5) the rest of algorithms for Section 3.3 and Section 3.4 are identical.

In the following, Lemma 4, we encapsulate this framework, bounding the performance of projected (stochastic) subgradient descent to the Lovasz extension, i.e. applying Theorem 3 to $\hat{f}$ over $\chi=[0,1]^{n}$. Formally, we abstract away the properties of a subgradient oracle data structure that we need to achieve a fast algorithm. With this lemma in place the remainder of the work in Section 3.2, Section 3.3, and Section 3.4 is to show how to efficiently implement the subgradient oracle in the particular setting.

Lemma 4. Suppose that there exists a procedure which maintains $\left(x^{(i)}, \tilde{g}^{(i)}\right)$ satisfying the invariants: (a) $\tilde{g}^{(i)}$ is $k$-sparse, (b) $\mathbf{E}\left[\tilde{g}^{(i)}\right]=g\left(x^{(i)}\right)$ is the Lovasz subgradient at $x^{(i)},(c) \mathbf{E}\left\|\tilde{g}^{(i)}\right\|_{2}^{2} \leq B^{2}$. Furthermore, suppose given any $e^{(i)}$ which is $k$-sparse, the procedure can update to $\left(x^{(i+1)}=x^{(i)}+e^{(i)}, \tilde{g}^{(i+1)}\right)$ in time $\mathrm{T}_{\mathrm{g}}$. Then, for any $\varepsilon>0$, we can compute a set $S$ with $\mathbf{E}[f(S)] \leq$ opt $+\varepsilon$ in time $O\left(n B^{2} \varepsilon^{-2} \mathrm{~T}_{\mathrm{g}}+n \mathrm{EO}+n \log n\right)$. If invariants (b) and (c) hold without expectation, then so does our algorithm.

Proof. We invoke Theorem 3 on the convex function $\hat{f}:[0,1]^{n} \rightarrow \mathbb{R}$ over the convex domain $\chi=[0,1]^{n}$ to obtain the iterates where we use the given subgradient oracle. Clearly

$$
x^{(1)}=\operatorname{argmin}_{x \in[0,1]^{n}} \frac{1}{2}\|x\|_{2}^{2}=0 \in \mathbb{R}^{n}
$$

and

$$
R^{2}=\sup _{x \in[0,1]^{n}} \frac{1}{2}\|x\|_{2}^{2}=\frac{1}{2}\|1\|_{2}^{2}=\frac{n}{2} .
$$

Consequently, as long as we implement the projection step for $T=O\left(n B^{2} \varepsilon^{-2}\right)$ steps (each step requiring $\mathrm{T}_{\mathrm{g}}$ time), then Theorem 3 yields

$$
\mathbf{E} \hat{f}\left(\frac{1}{T} \sum_{i \in[T]} x^{(i)}\right)-\min _{x \in \chi} \hat{f}(x) \leq R B \sqrt{\frac{2}{T}} \leq \sqrt{\frac{n B^{2}}{T}} \leq \varepsilon .
$$


Furthermore, as we argued in Section 2.3 we can compute $S$ with $f(S) \leq \hat{f}\left(\frac{1}{T} \sum_{i \in[T]} x^{(i)}\right)$ in the time it takes to compute $\frac{1}{T} \sum_{i \in[T]} x^{(i)}$ plus additional $O(n \mathrm{EO}+n \log n)$ time. To prove the lemma all that remains to to reason about the complexity of computing the projection, i.e. $x^{(t+1)}$, given that all the subgradients we compute are $s$-sparse. However, since $x^{(t+1)}=\operatorname{argmin}_{x \in[0,1]^{n}} \| x-\left(x^{(t)}-\eta \tilde{g}\left(x^{(t)}\right) \|_{2}^{2}\right.$ decouples coordinate-wise - note that $x^{(t+1)}=\operatorname{median}\left\{0, x^{(t)}-\eta \tilde{g}\left(x^{(t)}\right), 1\right\}$, we subtract $\eta \tilde{g}\left(x^{(t)}\right)$ from $x^{(t)}$ and if any coordinate is less than 0 we set it to 0 and if any coordinate is larger than 1 we set it to 1 . Thus the edit vector $e^{(i)}$ is of sparsity $\leq k$. Combining these facts yields the described running time.

\subsection{Subgradients of the Lovasz Extension}

Here we provide structural results of submodular function that we leverage to compute subgradients of submodular functions in $o(n)$ time on average. First, in Lemma 5 we state a result due to Jegelka and Bilmes [30](also Hazan and Kale [24]) which puts an upper bound on the $\ell_{1}$ norm of subgradients of the Lovasz extension provided we have an upper bound on the maximum absolute value of the function. We provide a short proof for completeness.

Lemma 5 (Subgradient Upper Bound). If $|f(S)| \leq M$ for all $S \subseteq[n]$, then $\|g(x)\|_{1} \leq 3 M$ for all $x \in[0,1]^{n}$ and for all subgradients $g$ of the Lovasz extension.

Proof. For notational simplicity suppose without loss of generality (by changing the name of the coordinates) that $P(x)=(1,2, \ldots, n)$, i.e. $x_{1} \geq x_{2} \geq \ldots \geq x_{n}$. Therefore, for any $i \in[n]$,we have $g_{i}=f([i])-f([i-1])$. Let $r_{1} \leq r_{2} \leq \ldots, \leq r_{R}$ denote all the coordinates such that $g_{r_{i}}>0$ and let $s_{1} \leq s_{2} \leq \ldots \leq s_{S}$ denote all the coordinates such that $g_{s_{i}}<0$.

We begin by bounding the contribution of the positive coordinate, the $g_{r_{i}}$, to the norm of the gradient, $\|g\|_{1}$. For all $k \in[R]$ let $R_{k} \stackrel{\text { def }}{=}\left\{r_{1}, \ldots, r_{k}\right\}$ with $R_{0}=\emptyset$. By assumption we know that that $f\left(R_{0}\right)=\emptyset$. Furthermore, by submodularity, i.e. diminishing marginal returns, we know that for all $i \in[R]$

$$
f\left(R_{i}\right)-f\left(R_{i-1}\right) \geq f\left(\left[r_{i}\right]\right)-f\left(\left[r_{i}-1\right]\right)=: g_{r_{i}}=\left|g_{r_{i}}\right|
$$

Consequently $f\left(R_{R}\right)-f\left(R_{0}\right)=\sum_{i \in[R]} f\left(R_{i}\right)-f\left(R_{i-1}\right) \geq \sum_{i \in[R]}\left|g_{r_{i}}\right|$. Since $f\left(R_{0}\right)=0$ and $f\left(R_{R}\right) \leq M$ by assumption we have that $\sum_{i \in[k]}\left|g_{r_{i}}\right| \leq M$.

Next, we bound the contribution of the negative coordinatess, the $g_{s_{i}}$, similarly. For all $k \in[S]$ let $S_{k} \stackrel{\text { def }}{=}$ $\left\{s_{1}, \ldots, s_{k}\right\}$ with $S_{0}=\emptyset$. By assumption we know that that $f\left(S_{0}\right)=\emptyset$. Define $V:=[n] \backslash S$. Note that for all $i \in[S]$, the set $V \cup S_{i-1}$ is a superset of $\left[s_{i}-1\right]$. Therefore, submodularity gives us for all $i \in[S]$,

$$
f\left(V \cup S_{i}\right)-f\left(V \cup S_{i-1}\right) \leq f\left(\left[s_{i}\right]\right)-f\left(\left[s_{i}-1\right]\right)=g_{s_{i}}=-\left|g_{s_{i}}\right|
$$

Summing over all $i$, we get $f([n])-f(V) \leq \sum_{i \in[S]}-\left|g_{s_{i}}\right|$. Since $f([n]) \geq-M$ and $f(V) \leq M$ we have that $\sum_{i \in[S]}\left|g_{s_{i}}\right| \leq 2 M$. Combining these yields that $\|g\|_{1}=\sum_{i \in[n]}\left|g_{i}\right|=\sum_{i \in[R]}\left|g_{r_{i}}\right|+\sum_{i \in[S]}\left|g_{s_{i}}\right| \leq 3 M$.

Next, in Lemma 6 we provide a simple but crucial monotonicity property of the subgradient of $\hat{f}$. In particular we show that if we add (or remove) a positive vector from $x \in[0,1]^{n}$ to obtain $y \in[0,1]^{n}$ then the gradients of the untouched coordinates all decrease (or increase).

Lemma 6 (Subgradient Monotonicity). Let $x \in[0,1]^{n}$ and let $d \in \mathbb{R}_{\geq 0}^{n}$ be such that $y=x+d$ (resp. $y=x-d)$. Let $S$ denote the non-zero coordinates of $d$. Then for all $i \notin S$ we have $g(x)_{i} \geq g(y)_{i}$ (resp. $\left.g(x)_{i} \leq g(y)_{i}\right)$.

Proof. We only prove the case of $y=x+d$ as the proof of the $y=x-d$ case is analagous. Let $P^{(x)}$ and $P^{(y)}$ be the permutations consistent with $x$ and $y$. Note that $P^{(y)}$ can be obtained from $P^{(x)}$ by moving a subset of 
elements in $S$ to the left, and the relative ordering of elements not in $S$ remains the same. Therefore, for any $i \notin S$, if $r$ is its rank in $P^{(x)}$, that is, $P_{r}^{(x)}=i$, and $r^{\prime}$ is its rank in $P^{(y)}$, then we must have $P^{(y)}\left[r^{\prime}\right] \supseteq P^{(x)}[r]$. By submodularity, $\left.g(y)_{i}=f\left(P^{(y)}\left[r^{\prime}\right]\right)-f\left(P^{(y)}\left[r^{\prime}-1\right]\right) \leq f\left(P^{(x)}[r]\right)-f\left(P^{(x)}[r-1]\right)\right)=g(x)_{i}$.

Lastly, we provide Lemma 7 giving a simple formula for the sum of multiple coordinates in the subgradient.

Lemma 7 (Subgradient Intervals). Let $x \in[0,1]^{n}$ and let $P$ be the permutation consistent with $x$. For any positive integers $a \leq b$, we have $\sum_{i=a}^{b} g(x)_{P_{i}}=f(P[b])-f(P[a-1])$.

Proof. This follows immediately from the definition of $g(x): \sum_{i=a}^{b} g(x)_{P_{i}}=\sum_{i=a}^{b}(f(P[i])-f(P[i-1]))=$ $f(P[b])+\sum_{i=a}^{b-1} f(P[i])-\sum_{i=a}^{b-1} f(P[i])-f(P[a-1])$.

\subsection{Nearly Linear in $n$, Pseudopolynomial Time Algorithm}

Here we provide the first nearly linear time pseudopolynomial algorithm for submodular function minimization. Throughout this section we assume that our submodular function $f$ is integer valued with $|f(S)| \leq M$ for all $S \subseteq[n]$. Our goal is to deterministically produce an exact minimizer of $f$. The primary result of this section is showing the following, that we can achieve this in $\tilde{O}\left(n M^{3} \mathrm{EO}\right)$ time:

Theorem 8. Given an integer valued submodular function $f$ with $|f(S)| \leq M$ for all $S \subseteq[n]$ in time $O\left(n M^{3} \mathrm{EO} \log n\right)$ we can compute a minimizer of $f$.

We prove the theorem by describing $\left(x^{(i)}, \tilde{g}\left(x^{(i)}\right)\right)$ in Lemma 4. In fact, in this case, $\tilde{g}$ will deterministically be the subgradient of the Lovasz extension. In Lemma 9, we prove that the Lovasz subgradient is $O(M)$ sparse and so $\|g\|_{2}^{2} \leq O\left(M^{2}\right)$. Thus, Conditions (a), (b), and (c) are satisfied with $B^{2}=O\left(M^{2}\right)$. The main contribution of this section is Lemma 10 , where we show that $\mathrm{T}_{\mathrm{g}}=O(M \log n \cdot \mathrm{EO})$, that is the subgradient can be updated in this much time. A pseudocode of the full algorithm can be found in Section E.

Lemma 9. For integer valued $f$ with $|f(S)| \leq M$ for all $S$ the subgradient $g(x)$ has at most $3 M$ non-zero entries for all $x \in[0,1]^{n}$.

Proof. By Lemma 5 we know that $\|g(x)\|_{1} \leq 3 M$. However, since $g(x)_{P_{i}}=f(P[i])-f(P[i-1])$ and since $f$ is integer valued, we know that either $g(x)_{P_{i}}=0$ or $\left|g(x)_{P_{i}}\right| \geq 1$. Consequently, there are at most $3 M$ values of $i$ for which $g(x)_{i} \neq 0$.

Lemma 10. With $O(n \cdot \mathrm{EO})$ preprocessing time the following data structure can be maintained. Initially, one is input $x^{(0)} \in[0,1]^{n}$ and $g\left(x_{0}\right)$. Henceforth, for all $i$, given $g\left(x^{(i)}\right)$ and a vector $e^{(i)}$ which is $k$-sparse, in $O(k \log n+k \mathrm{EO}+M \mathrm{EO} \log n)$ time one can update $g\left(x^{(i)}\right)$ to the gradient $g\left(x^{(i+1)}\right)$ for $x^{(i+1)}=x^{(i)}+e^{(i)}$.

Proof. The main idea is the following. Suppose $e^{(i)}$ is non-negative (we later show how to easily reduce to the case where all coordinates in $e^{(i)}$ have the same sign and the non-positive case is similar) Thus, by Lemma 6, for all coordinates not in support of $e^{(i)}$, the gradient goes down. Due to Lemma 9, the total number of change is $O(M)$, and since we can evaluate the sum of gradients on intervals by Lemma 7, a binary search procedure allows us to find all the gradient changes in $O(M \log n \cdot \mathrm{EO})$ time. We now give full details of this idea.

We store the coordinates of $x^{(i)}$ in a balanced binary search tree (BST) with a node for each $j \in[n]$ keyed by the value of $x_{j}^{(i)}$; ties are broken consistently, e.g. by using the actual value of $j$. We take the order of the nodes $j \in[n]$ in the binary search tree to define the permutation $P^{(i)}$ which we also store explicitly in a link-list, so we can evaluate $f\left(P^{(i)}[k]\right)$ in $O(\mathrm{EO})$ time for any $k$. Note that each node of the BST corresponds to a subinterval of $P^{(i)}$ given by the children of that node in the tree. At each node of the BST, we store the sum of $g\left(x^{(i)}\right)_{j}$ for all children $j$ of that node, and call it the value of the node. Note by Lemma 7 each 
individual such sum can be computed with 2 calls to the evaluation oracle. Finally, in a linked list, we keep all indices $j$ such that $g\left(x^{(i)}\right)_{j}$ is non-zero and we keep pointers to them from their corresponding node in the binary search tree. Using the binary search tree and the linked list, one can clearly output the subgradient. Also, given $x^{(0)}$, in $O(n \cdot \mathrm{EO})$ time one can obtain the initialization. What remains is to describe the update procedure.

We may assume that all non-zero entries of $e^{(i)}$ are the same sign; otherwise write $e^{(i)}:=e_{+}^{(i)}+e_{-}^{(i)}$, and perform two updates. WLOG, lets assume the sign is + (the other case is analogous). Let $S$ be the indices of $e^{(i)}$ which are non-zero.

First, we change the key for each $j \in[n]$ such that $x_{j}^{(i+1)} \neq x_{j}^{(i)}$ and update the BST. Since we chose a consistent tie breaking rule for keying, only these elements $j \in[n]$ will change position in the permutation $P^{(i+1)}$. Furthermore, performing this update while maintaining the subtree labels can be done in $O(k \log n)$ time as it is easy to see how to implement binary search trees that maintain the subtree values even under rebalancing. For the time being, we retain the old values as is.

For brevity, let $g^{(i)}$ and $g^{(i+1)}$ denote the gradients $g\left(x^{(i)}\right)$ and $g\left(x^{(i+1)}\right)$, respectively. Since we assume all non-zero changes in $e^{(i)}$ are positive, by Lemma 6 , we know that $g_{j}^{(i+1)} \leq g_{j}^{(i)}$ for all $j \notin S$. First, since $|S| \leq k$, for all $j \in S$, we go ahead and compute $g_{j}^{(i+1)}$ in $O(k \mathrm{EO})$ time. For each such $j$ we update the value of the nodes from $j$ to the root, by adding the difference $\left(g_{j}^{(i+1)}-g_{j}^{(i)}\right)$ to each of them. Next, we perform the following operation top-down start at the root: at each node we compare the current subtree value stored at this node with what the value actually should be with $g^{(i+1)}$. Note that since we know $P^{(i+1)}$, the latter can be computed with 2 evaluation queries. The simple but crucial observation is that if at any node $j$ these two values match, then we are guaranteed that $g_{k}^{(i+1)}=g_{k}^{(i)}$ for all $k$ in the tree rooted at $j$ and we do not need to recurse on the children of this node. The reason for equality is that for all the children, we must have $g_{k}^{(i+1)} \leq g_{k}^{(i)}$ by Lemma 6 , and so if the sum is equal then we must have equality everywhere. Since there are at most $O(M)$ coordinates change, this takes $O(M \mathrm{EO} \log n)$ for updating all the changes to $g^{(i+1)}$ for the binary search tree. During the whole process, whenever a node changes from non-zero to zero or from zero to non-zero, we can update the linked-list accordingly.

Proof of Theorem 8. We apply Lemma 4 giving the precise requirements of our subgradient oracle. We know that the subgradients we produce are always $O(M)$ sparse by Lemma 9 and satisfy $B^{2}=O\left(M^{2}\right)$. Consequently, we can simply instantiate Lemma 10 with $k=O(M)$ to obtain our algorithm. Furthermore, since $f$ is integral we know that so long as we have a set additive error less than 1 , i.e. $\epsilon<1$, the set is a minimizer. Consequently, we can minimize in the time given by the cost of adding the cost of Lemma 2, with the Lemma 9 initialization cost, plus the Lemma 9 cost for $T=O\left(n M^{2}\right)$ iterations, yielding

$$
O\left(n\left(\mathrm{EO}+\log n+M^{3}\right)+n+M \mathrm{EO}+(M \log n+M \mathrm{EO} \log n) \cdot n M^{2}\right)=O\left(n M^{3} \mathrm{EO} \log n\right) .
$$

\subsection{Subquadratic Additive Approximation Algorithm}

Here we provide the first subquadratic additive approximation algorithm for submodular function minimization. Throughout this section we assume that $f$ is real valued with $|f(S)| \leq 1$ for all $S \subseteq[n]$. Our goal is to provide a randomized algorithm that produces a set $S \subseteq[n]$ such $\mathbf{E} f(S) \leq$ opt $+\epsilon$. The primary result of this section is showing the following, that we can achieve this in $O\left(n^{5 / 3} \epsilon^{-2} \log ^{4} n\right)$ time:

Theorem 11. Given a submodular function $f: 2^{[n]} \rightarrow \mathbb{R}$ with $|f(S)| \leq 1$ for all $S \subseteq V$, and any $\varepsilon>0$, we we can compute a random set $S$ such that $\mathbf{E} f(S) \leq$ opt $+\epsilon$ in time $O\left(n^{5 / 3} \epsilon^{-2} \mathrm{EO} \log ^{4} n\right)$.

The proof of this theorem has two parts. Note that the difficulty in the real-valued case is that we can no longer assume the Lovasz gradients are sparse, and so we cannot do naive updates. Instead, we use the fact 
that the gradient has small $\ell_{2}$ norm to get sparse estimates of the gradient. This is the first part where we describe a sampling procedure which given any point $x$ and a $k$-sparse vector $e$, returns a good and sparse estimate to the difference between the Lovasz gradient at $x+e$ and $x$. The second issue we need to deal with is that if we naively keep using this estimator, then the error (variance) starts to accumulate. The second part then shows how to use the sampling procedure in a "batched manner" so as to keep the total variance under control, restarting the whole procedure with a certain frequency. A pseudocode of the full algorithm can be found in Section E.

Lemma 12. Suppose a vector $x \in[0,1]^{n}$ is stored in a BST sorted by value. Given a $k$-sparse vector e which is either non-negative or non-positive, and an integer $\ell \geq 1$, there is a randomized sampling procedure which returns a vector $z$ with the following properties: (a) $\mathbf{E}[z]=g(x+e)-g(x)$, (b) $\mathbf{E}\left[\|z-\mathbf{E}[z]\|_{2}^{2}\right]=O(1 / \ell)$, and (c) the number of non-zero coordinates of $z$ is $O(\ell)$. The time taken by the procedure is $O\left((k+\ell) \cdot \mathrm{EO} \log ^{2} n\right)$.

Proof. We assume that each non-zero value of $e$ is positive as the other case is analogous. Note that $x$ is stored in a BST, and the permutation $P_{x}$ consistent with $x$ is stored in a doubly linked list. Let $S$ be the set of positive coordinates of $e$ with $|S|=k$ and let $y$ denote the vector $x+e$. We compute $P_{y}$ in $O(k \log n)$ time.

Let $I_{1}, \ldots, I_{2 k} \subseteq[n]$ denote the subsets of the coordinates that correspond to the intervals which are contiguous in both $P_{x}$ and $P_{y}$. Note that these are $\leq 2 k$ such intervals, and some of them can be empty. We store the pointers to the endpoints of each interval in the BST. This can be done in $O(k \log n)$ time as follows. First compute the coarse intervals which are contiguous in $P_{x}$ in $O(k)$ time. These intervals will be refined when we obtain $P_{y}$. In $O(k \log n)$ time, update the BST so that for every node we can figure out which coarse interval it lies in $O(\log n)$ time. This is done by walking up the BST for every end point of all the $k$ intervals and storing which "side" of the interval they lie in. Given a query node, we can figure out which interval it lies in by walking up the BST to the root. Finally, for all nodes in $S$, when we update the BST in order to obtain $P_{y}$, using the updated data structure in $O(\log n)$ time figure out which coarse interval it lies in and refine that interval.

For each $j \in S$, we compute $d_{j} \stackrel{\text { def }}{=} g(y)_{j}-g(x)_{j}$ explicitly. This can be done in $O(k \mathrm{EO})$ time using $P_{x}$ and $P_{y}$.For $r \in[2 k]$, we define $D_{r}:=\sum_{j \in I_{r}}\left(g(y)_{j}-g(x)_{j}\right)$. Since each $I_{r}$ is a contiguous interval in both $P_{x}$ and $P_{y}$, Lemma 7 implies that we can store all $D_{r}$ in $O(k \cdot \mathrm{EO})$ time in look-up tables. Note that by monotonicity Lemma 6 each summand in $D_{r}$ is of the same sign, and therefore summing the absolute values of $D_{r}$ 's and $d_{j}$ 's gives $\|g(y)-g(x)\|_{1}$. We store this value of the $\ell_{1}$ norm.

Now we can state the randomized algorithm which returns the vector $z$. We start by sampling either a coordinate $j \in S$ with probability proportional to $\left|d_{j}\right|$, or an interval $I_{r}$ with probability proportional to $\left|D_{r}\right|$. If we sample an interval, then iteratively sample sub-intervals $I^{\prime} \subset I_{r}$ proportional to $\sum_{j \in I^{\prime}}\left(g(y)_{j}-g(x)_{j}\right)$ till we reach a single coordinate $j \notin S$. Note that any $j \in[n]$ is sampled with probability proportional to $\left|g(y)_{j}-g(x)_{j}\right|$.

We now show how to do this iterative sampling in $O((\mathrm{EO}+\log n) \log n)$ time. Given $I_{r}$, we start from the root of the BST and find a node closest to the root which lies in $I_{r}$. More precisely, since for every ancestor of the endpoints of $I_{r}$, if it doesn't belong to the interval we store which "side" of the tree $I_{r}$ lies in, one can start from the root and walk down to get to a node inside $I_{r}$. This partitions $I_{r}$ into two subintervals and we randomly select $I^{\prime}$ proportional to $\sum_{j \in I^{\prime}}\left(g(y)_{j}-g(x)_{j}\right)$. Since sub-intervals are contiguous in $P_{y}$ and $P_{x}$, this is done in $O(\mathrm{EO})$ time. We then update the information at every ancestor node of the endpoints of the sampled $I^{\prime}$ in $O(\log n)$ time. Since each iteration decreases the height of the least common ancestor of the endpoints of $I^{\prime}$, in $O(\log n)$ iterations (that is the height of the tree), we will sample a singleton $j \notin S$.

In summary, we can sample $j \in[n]$ with probability proportional to $g(y)_{j}-g(x)_{j}$ in $O((\mathrm{EO}+\log n) \log n)$ time. If we sample $j$, we return the (random) vector

$$
z:=\|g(y)-g(x)\|_{1} \cdot \operatorname{sign}\left(g(y)_{j}-g(x)_{j}\right) \cdot \mathbf{1}_{j}
$$


where recall $\mathbf{1}_{j}$ is the vector with 1 in the $j$ th coordinate and zero everywhere else. Note that given $j$, computing $z$ takes $O(\mathrm{EO}+\log n)$ time since we have to evaluate $g(y)_{j}$ and $g(x)_{j}$. Recall, we already know the $\ell_{1}$ norm. Also note by construction, $\mathbf{E}[z]$ is precisely the vector $g(y)-g(x)$. To upper bound the variance, note that

$$
\mathbf{E}\left[\|z-\mathbf{E} z\|_{2}^{2}\right] \leq \mathbf{E}\left[\|z\|_{2}^{2}\right]=\|g(y)-g(x)\|_{1}^{2} \leq 9 \cdot \max _{S \subseteq V}|f(S)| \leq 9
$$

by Lemma 5 and the fact that $|f(S)| \leq 1$. Also observe that $z$ is 1 -sparse.

Given $\ell$, we sample independently $\ell$ such random $z$ 's and return their average. The expectation remains the same, but the variance scales down by $\ell$. The sparsity is at most $\ell$. The total running time is $O(k(\mathrm{EO}+$ $\log n)+\ell(\mathrm{EO}+\log n) \log n)$. This completes the proof of the lemma.

We now complete the proof of Theorem 11.

Proof. (Theorem 11) The algorithm runs in batches (as mentioned before, the pseudocode is in Section E.) At the beginning of each batch, we have our current vector $x^{(0)}$ as usual stored in a BST. We also compute the Lovasz gradient $g^{(0)}=g\left(x^{(0)}\right)$ spending $O(n \log n \mathrm{EO})$ time. The batch runs for $T=\Theta\left(n^{1 / 3}\right)$ steps. At each step $t \in[T]$, we need to specify an estimate $\tilde{g}^{(t)}$ to run the (stochastic) subgradient procedure as discussed in Lemma 4. For $t=0$, since we know $g^{(0)}$ explicitly, we get $\tilde{g}^{(0)}$ by returning $\left\|g^{(0)}\right\|_{1} \operatorname{sign}\left(g_{j}^{(0)}\right) \mathbf{1}_{\mathbf{j}}$ with probability proportional to $\left|g_{j}^{(0)}\right|$. This is a 1-sparse, unbiased estimator of $g^{(0)}$ with $O(1)$ variance. Define $z^{(0)}:=\tilde{g}^{(0)}$. Henceforth, for every $t \geq 0$, the subgradient descent step suggests a direction $e^{(t)}$ in which to move whose sparsity is at most the sparsity of $\tilde{g}^{(t)}$. We partition $e^{(t)}=e_{+}^{(t)}+e_{-}^{(t)}$ into its positive and negative components. We then apply Lemma 12 twice: once with $x=x^{(t)}, e=e_{+}^{(t)}$, and $\ell=t$, to obtain random vector $z_{+}^{(t)}$ of sparsity $t$, and then with $x=x^{(t)}+e_{+}^{(t)}, e=e_{-}^{(t)}$, and $\ell=t$, to obtain the random vector $z_{-}^{(t)}$ of sparsity $t$. The estimate of the gradient at time $t$ is the sum of these random vectors. That is, for all $t \geq 1$, define $\tilde{g}^{(t)}:=\sum_{s \leq t}\left(z_{+}^{(s)}+z_{-}^{(s)}\right)$. By the property (b) of Lemma $12, \tilde{g}^{(t)}$ is a valid stochastic subgradient and can be fed into the framework of Lemma 4. Note that for any $t \in[T]$, the sparsity of $\tilde{g}^{(t)}$ is $O\left(t^{2}\right)$ and so is the sparsity of $e^{(t)}$ suggested by the stochastic subgradient routine. Thus, the $t$ th step of estimating $z_{+}^{(t)}$ and $z_{-}^{(t)}$ requires time $O\left(t^{2} \mathrm{EO} \log ^{2} n\right)$, implying we can run $T$ steps of the above procedure in $O\left(T^{3} \mathrm{EO} \log ^{2} n\right)$ time.

Finally, to argue about the number of iterations required to get $\varepsilon$-close, we need to upper bound $\mathbf{E}\left[\left\|\tilde{g}^{(t)}\right\|_{2}^{2}\right]$ for every $t$. Since $\mathbf{E}\left[\tilde{g}^{(t)}\right]=g^{(t)}$, the true subgradient at $x^{(t)}$ and since $\left\|g^{(t)}\right\|_{2}^{2}=O(1)$ by Lemma 5 , it suffices to upper bound $\mathbf{E}\left[\left\|\tilde{g}^{(t)}-\mathbf{E}\left[\tilde{g}^{(t)}\right]\right\|_{2}^{2}\right]$. But this follows since $\tilde{g}^{(t)}$ is just a sum of independent $z$-vectors.

$$
\mathbf{E}\left[\left\|\tilde{g}^{(t)}-\mathbf{E}\left[\tilde{g}^{(t)}\right]\right\|_{2}^{2}\right]=\sum_{s \leq t} \mathbf{E}\left[\left\|z_{+}^{(s)}-\mathbf{E}\left[z_{+}^{(s)}\right]\right\|_{2}^{2}\right]+\sum_{s \leq t} \mathbf{E}\left[\left\|z_{-}^{(s)}-\mathbf{E}\left[z_{-}^{(s)}\right]\right\|_{2}^{2}\right]=O\left(\sum_{s \leq t} 1 / s\right)=O(\log n)
$$

The second-last inequality follows from (c) of Lemma 12. And so, $\mathbf{E}\left[\left\|\tilde{g}^{(t)}\right\|_{2}^{2}\right]=\mathbf{E}\left[\left\|\tilde{g}^{(t)}-\mathbf{E}\left[\tilde{g}^{(t)}\right]\right\|_{2}^{2}\right]+\left\|g^{(t)}\right\|_{2}^{2}=$ $O(\log n)$. Therefore, we can apply the framework in Lemma 4 with $B=O(\log n)$ implying the total number of steps to get $\varepsilon$-approximate is $N=O\left(n \log ^{2} n \varepsilon^{-2}\right)$. Furthermore, since each batch takes time $O\left(\left(n+T^{3}\right) \mathrm{EO} \log ^{2} n\right)$ and there are $N / T$ batches, we get that the total running time is at most

$$
O\left(n \mathrm{EO} \log ^{4} n \varepsilon^{-2}\left(\frac{n+T^{3}}{T}\right)\right)=\tilde{O}\left(n^{5 / 3} \varepsilon^{-2} \mathrm{EO}\right)
$$


if $T=n^{1 / 3}$. This ends the proof of Theorem 11 .

\subsection{Improvements when Minimizer is Sparse}

Here we discuss how to improve our running times when the submodular function $f$ is known to have a sparse solution, that is, the set minimizing $f(S)$ has at most $s$ elements. Throughout this section we suppose we know $s$.

Theorem 13. Let $f$ be a submodular function with a s-sparse minimizer. Then if $f$ is integer valued with $|f(S)| \leq M$ for all $S \subseteq[n]$ we can compute the minimizer deterministically in time $O\left(\left(n+s M^{3}\right) \log n \cdot \mathrm{EO}\right)$. Furthermore if $f$ is real valued with $|f(S)| \leq 1$ for all $S \subseteq[n]$, then there is a randomized algorithm which in time $\tilde{O}\left(\left(n+s n^{2 / 3}\right) \mathrm{EO} \varepsilon^{-2}\right)$ returns a set $S$ such that $\mathbf{E}[f(S)] \leq$ opt $+\varepsilon$, for any $\varepsilon>0$.

Therefore, if we know that the sparsity of the optimum solution is, say polylog $(n)$, then there is a near linear time approximate algorithm to get constant additive error.

To obtain this running time we leverage the same data structures for maintaining subgradients presented in Section 3.3 and Section 3.4. Instead we show how to specialize the framework we used presented in Section 3.1. In particular we simply leverage that rather than minimizing the Lovasz extension over $[0,1]^{n}$ we can minimize over $S_{s} \stackrel{\text { def }}{=}\left\{x \in[0,1]^{n} \mid \sum_{i \in[n]} x_{i} \leq s\right\}$. This preserves the value of the maximum and minimum, but now improves the convergence of projected (stochastic) subgradient descent (because the quantity $R$ becomes $s$ from $n$ ). To show this formally we simply need to show that the projection step doesn't hurt the performance of our algorithm asymptotically.

We break the proof of this into 3 parts. First, in Lemma 14 we compute how to projection onto $S_{s}$. Then in Lemma 15 we show how to update our framework. Using these, we prove Theorem 13.

Lemma 14. For $k \geq 0$ and $y \in \mathbb{R}^{n}$ let $S=\left\{x \in[0,1]^{n} \mid \sum_{i} x_{i} \leq k\right\}$ and

$$
z=\operatorname{argmin}_{x \in S} \frac{1}{2}\|x-y\|_{2}^{2} .
$$

Then, we have that for all $i \in[n]$

$$
z_{i}=\operatorname{median}\left(0, y_{i}-\lambda, 1\right)
$$

where $\lambda$ is the smallest non-negative number such that $\sum_{i} z_{i} \leq k$.

Proof. By the method of Lagrange multiplier, we know that there is $\lambda \geq 0$ such that

$$
z=\operatorname{argmin}_{x \in[0,1]^{n}} \frac{1}{2}\|x-y\|_{2}^{2}+\lambda \sum_{i \in[n]} x_{i} .
$$

Since each variable in this problem is decoupled with each other, we can solve this problem coordinate-wise and get that for all $i \in[n]$

$$
z_{i}=\operatorname{med}\left(0, y_{i}-\lambda, 1\right) \text {. }
$$

Since $\sum_{i \in[n]} z_{i}$ decreases as $\lambda$ increases, we know that $\lambda$ is the smallest non-negative number such that $\sum_{i \in[n]} z_{i} \leq k$.

In particular we provide Lemma 15 and improvement on Lemma 4.

Lemma 15. Suppose that for $N \geq s B^{2} \epsilon^{-2}$ and any sequence of $x^{(1)}, . ., x^{(N)}$ such that that $x^{(i+1)}-x^{(i)}$ is $k^{(i)}$-sparse up to modifications that do not affect the additive distance between non-zero coordinates with 
$K \stackrel{\text { def }}{=} \sum_{i \in[T]} k^{(i)}=O(N k)$ we can implement a subgradient oracle for $f$ and $x^{(i)}$, denoted $\tilde{g}\left(x^{(i)}\right)$, that is $k$-sparse and obeys $\mathbf{E}\|\tilde{g}\|_{2}^{2} \leq B^{2}$. Then in time $O(n(\mathrm{EO}+\log n)+N k \log n)$ we can compute a set $S$ such that $\mathbf{E} f(S) \leq \mathrm{opt}+\epsilon$ (and if the subgradient oracle is deterministic then the result holds without the expectation).

Proof. The proof is the same as before, just the size of $R$ improves to $s$ and we need to deal with this new projection step. However, in the projection step we set all the coordinates that are less than 0 to 0 and then keep subtracting uniformly (stopping whenever a coordinate reaches 0 ) until the maximum coordinate is $\leq 1$. We can do this efficiently by simply maintaining an additive offset and the coordinate values in sorted order. Then we simply need to know the number of coordinates above some threshold and the maximum and the minimum non-zero coordinate to determine what to subtract up to the point we make the minimum nonzero. We can do this in $O(\log n)$ easily. Now we are not counting the movements that do not set something to 0 so do not change the additive distances between the non-zero coordinate. Consequently, an iteration may only move many coordinates if it sets many things to 0 , however that is paid for by the movement that created it, so we only need $N k \log n$ time in total to do all the updates.

We now have everything we need to prove Theorem 13

Proof of Theorem 13. (Sketch) The proof is the same as in Section 3.3 and Section 3.4. We just use Lemma 15 instead of the previous framework lemma. To invoke the first data structure just do the update in batches. the second data structure was already written for this setting.

\section{Lower Bound}

It is well known that $\Omega(n)$ evaluation oracle calls are needed to minimize a submodular function. On the other hand, the best way we know of for certifying minimality takes $\Theta(n)$ subgradient oracle calls (or equivalently, vertices of the base polyhedron). A natural question is whether $\Theta(n)$ subgradient oracle calls are in fact needed to minimize a submodular function. In this section we answer this in the affirmative. Since each gradient oracle needs $n$ evaluation oracle calls, this gives an $\Omega\left(n^{2}\right)$ lower bound on the number of evaluations required for algorithms which only access the function via graident oracles. As mentioned in the introduction, these include the Fujishige-Wolfe heuristic $[48,15]$, various version of conditional gradient or Franke Wolfe [14, 19] , and the new cutting plane methods [36]. Note that there are known lower bounds for subgradient descent that have a somewhat submodular structure [42] and this suggests that such a lower bound should be possible, however we are unaware of a previous information theoretic lower bound such as we provide.

To prove our lower bound, we describe a distribution over a collection of hard functions and show that any algorithm must make $\Omega(n)$ subgradient calls in expectation ${ }^{5}$ and by Yao's minimax principle this will give an $\Omega(n)$ lower bound on the expected query complexity of any randomized SFM algorithm. The distribution is the following. Choose $R$ to be a random set with each element of the universe selected independently with probability $1 / 2$. Given $R$, define the function

$$
f_{R}(S)= \begin{cases}-1 & \text { if } S=R \\ 0 & \text { if } S \subsetneq R \text { or } R \subsetneq S \\ 1 & \text { otherwise. }\end{cases}
$$

Clearly the minimizer of $f_{R}$ is the set $R$. Any SFM algorithm is equivalent to an algorithm for recognizing the set $R$ via subgradient queries to $f_{R}$. A subgradient $g$ of $f_{R}$ at any point $x$ corresponds to a permutation $P$ of $\{1,2, \ldots, n\}$ (the sorted order of $x$ ). Recall the notation $P[i]:=\left\{P_{1}, P_{2}, \ldots, P_{i}\right\}$. The following claim describes the structure of subgradients.

\footnotetext{
${ }^{5}$ One can also prove a high probability version of the same result but for simplicity we don't do it.
} 
Lemma 16. Let $i$ be the smallest index such that $P[i]$ is not a subset of $R$ and $j$ be the smallest index such that $P[j]$ is a superset of $R$. Then $g(i)=1, g(j)=-1$, and $g(k)=0$ for all $k \in[n] \backslash i, j$.

Proof. To see $g(i)=1$, note that $A:=P[i-1]$ is a subset of $R$. Two cases arise: either $A=R$ in which case $P[i]$ is a strict superset of $R$ and so $f_{R}(A)=-1$ and $f_{R}(P[i])=0$ implying $g(i)=1$; or $A$ is a strict subset of $R$ in which case $P[i]$ is neither a subset or a superset, implying $f_{R}(A)=0$ and $f_{R}(P[i])=1$. Similarly, to see $g[j]=-1$, note that $B:=P[j-1]$ is not a superset of $R$. Two cases arise: either $B$ is a strict subset of $R$ in which case $P[j]=R$ and we have $f_{R}(B)=0$ and $f_{R}(P[j])=-1$; or $B$ is neither a subset nor a superset in which case $P[j]$ is a strict superset of $R$ and we have $f_{R}(B)=1$ and $f_{R}(P[j])=0$.

For any other $k$, we have either both $P[k]$ and $P[k-1]$ are strict subsets of $R$ (if $k<\min (i, j)$ ), or both $P[k]$ and $P[k-1]$ are strict supersets of $R$ (if $k>\max (i, j)$ ), or both are neither superset nor subset. In all three cases, $g(k)=0$.

Intuitively, any gradient call gives the following information regarding $R$ : we know elements in $P[i-1]$ lie in $R, P_{i}$ doesn't lie in $R, P_{j}$ lies in $R$, and all $P_{k}$ for $k>j$ do not lie in $R$. Thus we get $i+n-j+1$ "bits" of information. If $R$ is random, then the expected value of this can be shown to be $O(1)$, and so $\Omega(n)$ queries are required. We make the above intuitive argument formal below.

Suppose at some point of time, the algorithm knows a set $A \subseteq R$ and a set $B \cap R=\emptyset$. The following lemma shows that one may assume wlog that subsequent subgradient calls are at points $x$ whose corresponding permutation $P$ contains the elements of $A$ as a "prefix" and elements of $B$ as a "suffix".

Lemma 17. Suppose we know $A \subseteq R$ and $B \cap R=\emptyset$. Let $g$ be a subgradient and $g^{\prime}$ be obtained from $g$ by moving $A$ and $B$ to the beginning and end of the permutation respectively. Then one can compute $g$ from $g^{\prime}$ without making any more oracle calls.

Proof. Easy by case analysis and Lemma 16. Let $P$ be the permutation corresponding to $g$. We show that given $g$ ' and $P$, we can evaluate $g$. Let us say we are interested in evaluating $g_{P_{k}}$ and say $P_{k}=a$. Lemma 16 states that this is 1 iff $P[k-1] \subseteq R$ and $P[k]$ isn't. Now, if $P[k-1] \cap B \neq \emptyset$, then we know $g_{P_{k}}=0$. Otherwise, $g_{P_{k}}=1$ iff $P[k-1] \backslash B \cup A \subseteq R$ and $P[k] \backslash B \cup A$ is not, since $A \subseteq R$. Therefore, $g_{P_{k}}=1$ iff $g_{a}^{\prime}=1$ and $P[k-1] \cap B=\emptyset$. Whether $g_{P_{k}}=-1$ or not can be done analogously.

For an algorithm, let $h(k)$ be the expected number of subgradient calls required to minimize $f_{R}$ when the universe if of size $k$ (note $R$ is chosen randomly by picking each element with probability $1 / 2$ ). For convenience we also define $h(k)=0$ for $k \leq 0$.

Lemma 18. For $k \geq 1, h(k) \geq 1+\mathbf{E}_{X, Y}[h(k-X-Y)]$, where $X, Y$ are independent geometric random variables, i.e. $\operatorname{Pr}[X=i]=1 / 2^{i}$ for $i \geq 1$.

Proof. By our observation above, a subgradient of $f$ reveals the identities of $\min \{X+Y, k\}$ elements, where $X-1=i-1$ and $Y-1=n-j(i, j$ as defined in Lemma 16) are the lengths of the streaks of 0 's at the beginning and end of the subgradient.

Note that $X$ simply follow a geometric distribution because $\operatorname{Pr}\left[P[i-1] \subseteq R, P_{i} \notin R\right]=1 / 2^{i}$. Similarly, $Y$ also follow the same geometric distribution. In the case of $X+Y>k$, we have $R$ as a prefix of the permutation.

Finally, as a subgradient call reveals no information about the intermediate elements in the permutation, by Lemma 17 we are then effectively left with the same problem of size $k-X-Y$. More formally, this is because the value of the subgradient queried is independent of the identities of the elements $P_{i+1}, \ldots, P_{j-1}$.

Theorem 19. $h(n) \geq n / 4$, i.e. any algorithm for SFM requires at least $\Omega(n)$ subgradient calls. 
Proof. We show by induction that $h(k) \geq k / 4$. By Lemma 18 and the induction hypothesis,

$$
\begin{aligned}
h(k) & \geq 1+\mathbf{E}_{X, Y}[h(k-X-Y)] \\
& \geq 1+\mathbf{E}_{X, Y}[(k-X-Y) / 4] \\
& =1+k / 4-\mathbf{E}[X] / 4-\mathbf{E}[Y] / 4 \\
& =k / 4
\end{aligned}
$$

as desired.

Readers may have noticed that the proofs of the preceding two lemmas essentially imply that $h(k)$ is roughly the expected number of geometric random variables needed to sum up to $k$. One can use this property together with some concentration inequality for geometric random variables to establish a high probability version of our lower bound.

\section{Acknowledgments}

This work was partially supported by NSF awards 0843915, 1111109, CCF0964033, CCF1408635 and Templeton Foundation grant 3966. Part of this work was done while the first three authors were visiting the Hausdorff Research Institute for Mathematics in Bonn for the Workshop on Submodularity, and the last three authors were visiting the Simons Institute for the Theory of Computing in Berkeley. We thank the organizers of the workshop for inviting us. We thank Elad Hazan and Dan Garber for helpful preliminary discussions regarding approximate SFM. We thank the anonymous reviewers for their helpful comments and in particular for pointing us to needed references and previous work as well as pointing us to the relationship between our work and graph optimization, encouraging us to write Appendix C. A special thanks to Bobby Kleinberg for asking the question about approximate SFM.

\section{References}

[1] Francis Bach. Learning with submodular functions: A convex optimization perspective. Foundations and Trends in Machine Learning, 6(2-3):145-373, 2013.

[2] Siddharth Barman. Approximating Nash equilibria and dense bipartite subgraphs via an approximate version of Caratheodory's theorem. ACM Symp. on Theory of Computing (STOC), 2015.

[3] Jeff Bilmes. Submodularity in machine learning applications. Twenty-Ninth Conference on Artificial Intelligence, AAAI-15 Tutorial Forum, January 2015.

[4] Yuri Boykov and Vladimir Kolmogorov. An experimental comparison of min-cut/max-flow algorithms for energy minimization in vision. IEEE Transactions on Pattern Analysis and Machine Intelligence (PAMI), 26(9):1124 - 1137, 2004.

[5] Yuri Boykov, Olga Veksler, and Ramin Zabih. Fast approximate energy minimization viagraph cuts. IEEE Transactions on Pattern Analysis and Machine Intelligence (PAMI), 23(11):1222 - 1239, 2001.

[6] Sébastien Bubeck. Convex optimization: Algorithms and complexity. Foundations and Trends in Machine Learning, 8(3-4):231-357, 2015.

[7] Niv Buchbinder, Moran Feldman, Seffi Naor, and Roy Schwartz. A tight linear time 1/2-approximation for unconstrained submodular maximization. SIAM J. Comput., 44(5):1384-1402, 2015. 
[8] Deeparnab Chakrabarty, Prateek Jain, and Pravesh Kothari. Provable submodular minimization via Fujishige-Wolfe algorithm. Adv. in Neu. Inf. Proc. Sys. (NIPS), 2014.

[9] Gustave Choquet. Theory of capacities. Annales de l'institut Fourier, 5:131-295, 1955.

[10] William Cunningham. On submodular function minimization. Combinatorica, 5:185 - 192, 1985.

[11] Jack Edmonds. Submodular functions, matroids and certain polyhedra. Combinatorial Structures and Their Applications, pages 69-87, 1970.

[12] Alina Ene and Huy L. Nguyen. Random coordinate descent methods forminimizing decomposable submodular functions. Proc, Int. Conf. on Machine Leanring (ICML), 2015.

[13] Uriel Feige, Vahab Mirrokni, and Jan Vondrak. Maximizing non-monotone submodular functions. SIAM J. Comput., 40(4):1133 - 1153, 2011.

[14] Marguerite Frank and Philip Wolfe. An algorithm for quadratic programming. Naval Research Logistics Quarterly, 3(1-2):95-110, 1956.

[15] Satoru Fujishige. Lexicographically optimal base of a polymatroid with respect to a weight vector. Math. Oper. Res., 5:186-196, 1980.

[16] Satoru Fujishige. Submodular functions and optimization. Elsevier, 2005.

[17] Satoru Fujishige, Takumi Hayashi, and Shigueo Isotani. The minimum-norm-point algorithm applied to submodular function minimization and linear programming. Publications of the Research Institute for Mathematical Sciences (RIMS), Kyoto, 2006.

[18] Satoru Fujishige and Shigueo Isotani. A submodular function minimization algorithm based on the minimum-norm base. Pacific Journal of Optimization, 7:3-17, 2011.

[19] Dan Garber and Elad Hazan. A linearly convergent variant of the conditional gradient algorithm under strong convexity, with applications to online and stochastic optimization. SIAM Journal on Optimization, 26(3):1493-1528, 2016.

[20] Michel X. Goemans and Jose A. Soto. Symmetric submodular function minimization under hereditary family constraints. 27(2):1123 - 1145, 2013.

[21] Martin Grotschel, Laszlo Lovasz, and Alexander Schrijver. The ellipsoid method and its consequences in combinatorial optimization. Combinatorica, 1:169 - 197, 1981.

[22] Nicholas James Alexander Harvey. Matchings, matroids and submodular functions. PhD thesis, Massachusetts Institute of Technology, 2008.

[23] Nick Harvey. Matroid intersection, pointer chasing, and Young's seminormal representation of $S_{n}$. Proceedings, ACM-SIAM Symposium on Discrete Algorithms (SODA), 2008.

[24] Elad Hazan and Satyen Kale. Online submodular minimization. J. Mach. Learn. Res., 13:2903 - 2922, 2012.

[25] Satoru Iwata, Lisa Fleischer, and Satoru Fujishige. A combinatorial strongly polynomial algorithm for minimizing submodular functions. J. ACM, 48(4):761-777, 2001.

[26] Satoru Iwata and James B. Orlin. A simple combinatorial algorithm for submodular function minimization. In SODA, pages 1230-1237, 2009.

[27] Rishabh Iyer and Jeff A. Bilmes. Submodular optimization with submodular cover and submodular knapsack constraints. Adv. in Neu. Inf. Proc. Sys. (NIPS), 2013. 
[28] Rishabh Iyer, Stefanie Jegelka, and Jeff A. Bilmes. Curvature and optimal algorithms for learning and minimizing submodular functions. Adv. in Neu. Inf. Proc. Sys. (NIPS), 2013.

[29] Stefanie Jegelka, Francis Bach, and Suvrit Sra. Reflection methods for user-friendly submodular optimization. Adv. in Neu. Inf. Proc. Sys. (NIPS), 2013.

[30] Stefanie Jegelka and Jeff Bilmes. Online submodular minimization for combinatorial structures. In ICML, pages 345-352, 2011.

[31] Stefanie Jegelka, Hui Lin, and Jeff A. Bilmes. On fast approximate submodular minimization. In NIPS, pages 460-468, 2011.

[32] Pushmeet Kohli, M. Pawan Kumar, and Philip H. S. Torr. P3 and beyond: Move making algorithms for solving higher order functions. IEEE Trans. Pattern Anal. and Machine Learning, 31, 2008.

[33] Pushmeet Kohli and Philip H. S. Torr. Dynamic graph cuts and their applications in computer vision. Computer Vision: Detection, Recognition and Reconstruction., 2010.

[34] Vladimir Kolmogorov. Minimizing a sum of submodular functions. Discrete Appl. Math., 160(15):2246 $-2258,2012$.

[35] S. Lacoste-Julien and M. Jaggi. On the global linear convergence of Frank-Wolfe optimization variants. In Adv. in Neu. Inf. Proc. Sys. (NIPS), 2015.

[36] Yin Tat Lee, Aaron Sidford, and Sam Chiu-Wai Wong. A faster cutting plane method and its implications for combinatorial and convex optimization. Proceedings, IEEE Symposium on Foundations of Computer Science (FOCS), 2015.

[37] Hui Lin and Jeff Bilmes. An application of the submodular principal partition to training data subset selection. NIPS workshop on Discrete Optimization in Machine Learning, 2010.

[38] Hui Lin and Jeff Bilmes. Optimal selection of limited vocabulary speech corpora. Proc. Ann. Conf. Int. Speech Comm. Ass. (INTERSPEECH), 2011.

[39] Laszlo Lovasz. Submodular functions and convexity. Mathematical Programming - The State of the Art. A. Bachem, M. Grotschel, B. Korte eds.,Springer, pages 235 - 257, 1983.

[40] S. T. McCormick. Submodular function minimization. Chapter 7 in the Handbook of Discrete Optimization, 2006.

[41] Vahab Mirrokni, Renato Paes Leme, Adrian Vladu, and Sam Chiu-Wai Wong. Tight bounds for approximate Caratheodory and beyond. arXiv, http://arxiv.org/abs/1512.08602, December, 2015.

[42] Yu Nesterov. Introductory Lectures on Convex Optimization: A Basic Course, volume I. 2003.

[43] Robert Nishihara, Stefanie Jegelka, and Michael I. Jordan. On the convergence rate of decomposable submodular function minimization. Adv. in Neu. Inf. Proc. Sys. (NIPS), 2014.

[44] Adarsh Prasad, Stefanie Jegelka, and Dhruv Batra. Submodular meets structured: Finding diverse subsets in exponentially-large structured item sets. In Adv. in Neu. Inf. Proc. Sys. (NIPS), 2014.

[45] Alexander Schrijver. A combinatorial algorithm minimizing submodular functions in strongly polynomial time. J. Comb. Theory, Ser. B, 80(2):346-355, 2000.

[46] Peter Stobbe and Andreas Krause. Efficient minimization of decomposable submodular functions. Adv. in Neu. Inf. Proc. Sys. (NIPS), 2010. 
[47] Zoya Svitkina and Lisa Fleischer. Submodular approximation: Sampling-based algorithms and lower bounds. SIAM J. Comput., 40, 2011.

[48] Philip Wolfe. Finding the nearest point in a polytope. Math. Programming, 11:128 - 149, 1976. 


\section{A Reduction from Multiplicative to Additive Approximation}

Here we show how to obtain a multiplicative approximation for SFM from our $\tilde{O}\left(n^{5 / 3} \cdot \mathrm{EO} / \varepsilon^{2}\right)$ additiveapproximate SFM algorithm. Because the minimizer of $f$ is scale- and additive-invariant, it is necessary to make certain regularity assumptions on $f$ to get a nontrivial result. This is akin to submodular function maximization where constant factor approximation is possible only if $f$ is nonnegative everywhere $[7,13]$. For SFM, by considering $f$ - opt we see that finding a multiplicative-approximate solution and an exact solution are equivalent for general $f$. (Indeed most submodular optimization problems permit multiplicative approximation only in terms of the range of values.)

Similar to submodular maximization, we assume $f$ to be nonpositive. Then $f^{\prime}=f$ opt has range $[-1,0]$ and has minimum value -1 so our additive-approximate algorithm immediately yields multiplicative approximation. This requires knowing opt (or some constant factor approximation of). Alternately we can "binary search" to get factor- 2 close to opt by trying different powers of 2 . This would lead to a blowup of $O(\log$ opt $)$ in the running time.

\section{B Approximate SFM via Fujishige-Wolfe}

Here we show how Frank-Wolfe and Wolfe can give $\varepsilon$-additive approximations for SFM. We know that both algorithms in $O(1 / \delta)$ iterations can return a point $x \in B_{f}$, the base polyhedron associated with $f$, such that $x^{\top} x \leq p^{\top} p+\delta$ for all $p \in B_{f}$. Here we are using the fact implied by Lemma 5 that the diameter of the base-polytope for functions with bounded range is bounded (note that vertices of the base polytope correspond to gradients of the Lovasz extension.) The robust Fujishige Theorem (Theorem 5, [8]) implies that we can get a set $S$ such that $f(S) \leq$ opt $+2 \sqrt{n \delta}$. Setting $\delta=\varepsilon^{2} / 4 n$ gives the additive approximation in $O\left(n \varepsilon^{-2}\right)$ gradient calls.

\section{Faster Algorithm for Directed Minimum Cut}

Here we show how to easily obtain faster approximate submodular minimization algorithms in the case where our function when the funciton is an explicitly given $s$ - $t$ cut function. This provides a short illustration of the reasonable fact that when given more structure, our sumbodular minimization algorithms can be improved. For the rest of this section, let $G=(V, E, w)$ be a graph with vertices $V$, directed edges $E \subseteq V \times V$, and edge weights $w \in \mathbb{R}_{\geq}^{E} 0$. Let $s, t \in V$ be two special vertices, $A \stackrel{\text { def }}{=} V \backslash\{s, t\}$, and for all $S \subseteq A$ let $f(S)$ be defined as the total weight of the edges in leaving the set $S \cup\{s\}$, i.e. where the tail of edge is in $S \cup\{s\}$ and the head of the edges is in $V \backslash(S \cup\{s\})$. The function $f$ is a well known submodular function and minimizing it corresponds to computing the minimum $s$ - $t$ cut, or correspondingly the maximum $s$ - $t$ flow.

Note that clearly, $f(S) \leq W$ where $W=\sum_{e \in E} w_{e}$. Furthermore, if we pick $S$ by including each vertex in $A$ randomly to be in $S$ with probability independently $\frac{1}{2}$ then we see that $\mathbf{E} f(S)=\frac{1}{2} W$. Consequently, $\frac{1}{2} W \leq \max _{S \subseteq A} f(s) \leq W$ and if we want to scale $f$ to make it have values in $[-1,1]$ we need to devide by something that is $W$ up to a factor of 2 .

Now, note that we can easily extend this problem to a continuous problem over the reals. Let $x^{+}$denote $x$ if $x \geq 0$ and 0 otherwise. Furthermore, for all $x \in \mathbb{R}^{A}$ let $y(x) \in \mathbb{R}^{V}$ be given by $y(x)_{i}=x_{i}$ if $i \in A, y(x)_{s}=0$, $y(x)_{t}=1$, and let

$$
g(x) \stackrel{\text { def }}{=} \sum_{(a, b) \in E} w_{a b}\left(y\left(x_{b}\right)-y\left(x_{a}\right)\right)^{+} .
$$

Clearly, minimizing $g(x)$ over $[0,1]^{A}$ is equivalent to minimizing $f(S)$. Furthermore the subgradient for $g$ decomposes into subgradients for each edge $(a, b) \in E$ each of which is a vector with 2 non-zero entries and 
norm at most $O\left(w_{a b}\right)$. If we picking a random edge with probability proportional to $w_{a b}$ and output its subgradient scaled by $W / w_{a b}$ subgradient this yields a stochastic subgradient oracle $\tilde{g}(x)$ with $\mathbf{E}\|\tilde{g}(x)\|_{2}^{2}=$ $O\left(\sum_{(a, b) \in E} \frac{w_{a b}}{W}\left(\left(W / w_{a b}\right) \cdot w_{a b}\right)^{2}\right)=O\left(W^{2}\right)$. Consequently, by Theorem 3 setting $R^{2}=O(|V|)$ we see that we can compute $z$ with $g(z)-\min _{x} g(x) \leq W \epsilon$ in $O\left(|v| \epsilon^{-2}\right)$. Thus, if we scaled $g$ to make it [-1,1] valued the time to compute an $\epsilon$-approximate solution would be $O\left(|V| \epsilon^{-2}\right)$.

This shows that an explicit instance of minimum $s$ - $t$ cut does not highlight the efficacy of the approach in this paper. Instantiating our algorithm naively would give an $\tilde{O}\left(|E| \cdot|V|^{5 / 3} \cdot \epsilon^{-2}\right)$ to achieve additive error $\epsilon$. Nevertheless, even for such an instance if instead we were simply given access to the an EO time evaluation oracle for $f$, and the graph was desne, even in this instance, without knowing the structure aprior we do not know how to improve upon the $O\left(\mathrm{EO} \cdot|V|^{5 / 3} \epsilon^{-2}\right)$ time bound achieved in this paper (though no serious attempt was made to do this). In short there may be a gap between explicitly given structured instances of submodular functions and algorithms that work with general evaluation oracles as focused on in this paper.

\section{Certificates for Approximate SFM}

The only certificate we know to prove that the optimum value of SFM is $\geq F$ is to show a certain vector $x$ lies in the base polyhedron. For example, one proof via Edmond's Theorem [11] is by demonstrating $x \in B_{f}$ whose negative entries sum to $\geq F$. The only way to do this is via Carathedeory's Theorem which requires $n$ vertices of $B_{f}$, each of which requires $n$ function evaluations. For approximate SFM, one thought might to be to use approximate Caratheodory's Theorems [2, 41] to describe a nearby point $x^{\prime}$. Unfortunately, for $\varepsilon$-additive SFM approximation, one needs $x^{\prime}$ and $x$ to be close in $\ell_{1}$-norm and approximate Caratheodory works only for $\ell_{2}$-norm and higher. If one uses the $\ell_{2}$-norm approximation, then unfortunately one doesn't get anything better than quadratic. More precisely, approximate Caratheodory states that one can obtain $\left\|x^{\prime}-x\right\|_{2} \leq \delta$ with support of $x^{\prime}$ being only $O\left(1 / \delta^{2}\right)$-sparse. But to get $\ell_{1}$ approximations, we need to set $\delta=\varepsilon \sqrt{n}$ leading to linear sized support for $x^{\prime}$. The approximate Caratheodory Theorems are tight [41] for general polytopes. Whether one can get better theorems for the base polyhedron is an open question.

\section{E Pseudocodes for Our Algorithms}

We provide guiding pseudocodes for our two algorithms. 
- $x^{(1)} \stackrel{\text { def }}{=} 0^{n}$

- Evaluate $g^{(1)}$ is the Lovasz subgradient at $x^{(1)}$. (Takes $O(n \cdot \mathrm{EO})$ time. Store as (coordinate, value) pair in set $S^{(1)} .\left|S^{(1)}\right| \leq 3 M$. )

- Store $x^{(1)}$ in a balanced Binary search tree. At each node store the value that is the sum of the gradient coordinates corr. to children in the tree. (Takes $O(n)$ time to build.)

- Set $T \stackrel{\text { def }}{=} 20 n M^{2}$. Set $\eta \stackrel{\text { def }}{=} \frac{\sqrt{n}}{18 M}$.

For $t=1,2, \ldots, T$ :

- Define $e^{(t)}$ which is non-zero in coordinates corresponding to $S^{(t)}$ : (Takes time $\left|S^{(t)}\right| \leq 3 M$.)

$$
\begin{aligned}
& \text { - if } g_{i}^{(t)}>0, \text { then } e_{i}^{(t)}=\min \left(x_{i}^{(t)}, \eta g_{i}^{(t)}\right) \\
& \text { - if } g_{i}^{(t)}<0, \text { then } e_{i}^{(t)}=\max \left(x_{i}^{(t)}-1, \eta g_{i}^{(t)}\right)
\end{aligned}
$$

- Update $\left(x^{(t)}, e^{(t)}, S^{(t)}\right)$ to get $\left(x^{(t+1)}, g^{(t+1)}, S^{(t+1)}\right)$ where $g^{(t+1)}$ is stored as coordinate, value pairs in $S^{(t+1)}$. as described in Lemma 10. (Update takes time $O(M \log n+M \cdot \mathrm{EO}+M \cdot \mathrm{EO} \log n)$ )

Obtain the $O(n)$ sets given the order of $x_{T}$, that is, if $P$ is the permutation corresponding to $x_{T}$, then the sets are $\{P[1], \ldots, P[n]\}$. Return the minimum valued set among them.

\footnotetext{
Algorithm 2 Subquadratic Approximate SFM Algorithm.

\section{Initialization}

- Set $N \stackrel{\text { def }}{=} 10 n \log ^{2} n \varepsilon^{-2}, T=\left\lceil n^{1 / 3}\right\rceil$

- Initialize $x$ as the all zeros vector and store it in a BST.

For $i=1,2, \ldots N / T$ :

- $x^{(1)} \stackrel{\text { def }}{=}$ the current $x$.

- Compute $g^{(1)}$, the gradient to the Lovasz extension given $x^{(1)}$. //This takes $O(n \mathrm{EO})$ time).

- Sample $z^{(1)}$ by picking $j \in[n]$ with probability proportional to $\left|g_{j}^{(1)}\right|$ and returning $z^{(1)} \stackrel{\text { def }}{=}$ $\left\|g^{(1)}\right\|_{1} \operatorname{sign}\left(g_{j}^{(1)}\right) \cdot \mathbf{1}_{j}$. //This takes $O(n \mathrm{EO})$ time.

- Set $\tilde{g}^{(1)} \stackrel{\text { def }}{=} z^{(1)}$.

- For $t=1,2, \ldots, T$ :

- Define $e^{(t)}$ as in Algorithm 1 using $\tilde{g}^{(t)}$ instead of $g^{(t)}$. //This takes time $O\left(\operatorname{supp}\left(\tilde{g}^{(t)}\right)\right)$ which will be $O\left(t^{2}\right)$

- Obtain $z^{(t)}$ using $\operatorname{Sample}\left(x^{(t)}, e^{(t)}, \ell=t\right)$ where Sample is the randomized procedure describe in Lemma 12. //This takes $O\left(t^{2} \mathrm{EO} \log n\right)$ time.

- Update $\tilde{g}^{(t+1)} \stackrel{\text { def }}{=} \sum_{s \leq t} z^{(s)}$. //This takes $O\left(t^{2} \log n\right)$ time to update the relevant BSTs.

- Set current $x$ to $x_{T}$.

Obtain the $O(n)$ sets given the order of the final $x$, that is, if $P$ is the permutation corresponding to $x$, then the sets are $\{P[1], \ldots, P[n]\}$. Return the minimum valued set among them.
} 